\title{
Applications of Cone Beam Computed Tomography in Orthodontics: An Overview
}

\author{
Prateeksha Bora*, Poonam Agrawal, Dinesh Kumar \\ Bagga, Sakshi Tiwari and Aartika Singh \\ ${ }^{1}$ Department of Orthodontics \& Dentofacial Orthopedics, School of Dental \\ Sciences, Sharda University, Greater Noida, Uttar Pradesh, India
}

\section{ABSTRACT}

Cone beam computerized tomography ( $\mathrm{CBCT}$ ) has changed numerous aspects of dentistry and added great value to its diagnostic phase also as that of orthodontics. Three-dimensional imaging CBCT has the potential to improve the diagnosis and treatment planning of cases. The introduction of cone beam computerized tomography (CBCT) technology to dentistry and orthodontics revolutionized the diagnosis, treatment and monitoring of orthodontic patients. The information obtained from CBCT imaging provides several substantial advantages. For example, CBCT imaging provides accurate measurements, improves localization of impacted teeth, provides visualization of airway abnormalities, it identifies and quantifies asymmetry, it are often wont to assess periodontal structures, to spot endodontic problems, to plan placement sites for temporary skeletal anchorage devices, and to look at condylar positions and mandibular joint (TMJ). Moreover, CBCT imaging involves only a minimal increase in radiation dose relative to combined diagnostic modern digital panoramic and cephalometric imaging. Cone-beam computed tomography (CBCT) is now being widely used in orthodontic practice. On the one hand, there is an increasing need of CBCT for diagnosis and research, and on the other hand, there is a broad concern for the exposure to radiation. Prescribing CBCT scan requires judicious clinical judgment to maximize the benefits and minimize the harm. The best way to reduce unnecessary ionizing radiation from CBCT is to follow recommended guidelines and ethical principles. CBCT provides a superb tool for accurate diagnosis, more predictable treatment planning, more efficient patient management and education, improved treatment outcome and patient satisfaction. This review discusses the utilization of CBCT in diagnosis and treatment planning in orthodontics.

KEY WORDS: CONE BEAM COMPUTED TOMOGRAPHY; CBCT; THREE-DIMENSIONAL IMAGING; ORTHODONTICS; EVIDENCE-BASED DIAGNOSIS; TREATMENT PLANNING AND TREATMENT OUTCOMES.

\section{INTRODUCTION}

Orthodontic clinical examination and documentation is the basis for diagnosis, treatment planning, growth evaluation, and outcome assessment in orthodontics.

\section{ARTICLE INFORMATION}

Received 30th Oct 2020 Accepted after revision 13th Dec 2020 Print ISSN: 0974-6455 Online ISSN: 2321-4007 CODEN: BBRCBA

Thomson Reuters ISI Web of Science Clarivate Analytics USA and Crossref Indexed Journal

\section{Clarivate
Analytics}

NAAS Journal Score 2020 (4.31)

A Society of Science and Nature Publication,

Bhopal India 2020. All rights reserved.

Online Contents Available at: http//www.bbrc.in/

Doi: http://dx.doi.org/10.21786/bbrc/13.15/27
Diagnostic records for an orthodontic patient consist of dental casts, intra- and extra-oral pictures, a panoramic $\mathrm{X}$-ray, and a lateral head film, supplemented with other diagnostic tests as indicated. Since the introduction of cone beam computerized tomography (CBCT) in 1998, there has been a rapid evolution in CBCT technology, driven largely by the stress of every specialty for providing accurate and reproducible three-dimensional (3D) images, and minimizing the radiation dose.

Applications of CBCT in orthodontics include evaluation of skeletal and dental malocclusions, localization of impacted canines and molars, evaluation of potential Temporary Anchorage sites, and for orthognathic surgical planning. CBCT also plays a valuable role in airway 
analysis and in managing syndromic patients, including cleft lip and palate. While the clinical applications spanning from evaluation of anatomy to pathology of most structures within the maxillo-facial area, the key advantage of CBCT is its high-resolution images at a comparatively lower radiation dose.

The main advantage of CBCT is that the ability to look at and analyse the osseous oral and maxillofacial structures in three dimensions (3D), overcoming the magnification and superimposition of structures found with two-dimensional (2D) imaging modalities. However, despite the advantages of CBCT imaging, it shouldn't be used routinely for all orthodontic patients, and there must be a risk- benefit balance between any radiation exposure versus the knowledge which will be gained by the investigation.

The introduction of CBCT specifically dedicated to imaging the maxillofacial region heralds a real paradigm shift. It has created a revolution in maxillofacial imaging expanding the role of imaging from diagnosis to image guidance of operative and surgical procedures with the assistance of varied software applications. Accurate diagnostic imaging is an important requirement to derive the right diagnosis and optimal treatment plan, also as monitor and document the treatment progress and outcome.

Essentials of CBCT Imaging: Cone-beam refers to the cone shape of the X-ray beam, unlike conventional CT, which uses a fan-shaped beam to create multiple thin slices. CBCT produces panoramic and cephalometric projections, which become 3D when the information is reformatted during a volume. With СBCT technology all possible radiographs are often taken in under 1 minute. The orthodontist now has the diagnostic quality of periapical, panoramic, cephalograms, occlusal radiographs, and TMJ series at their disposal, alongside views that can't be produced by regular radiographic machines like axial views, and separate cephalograms for the right and left sides. Clinicians are encouraged to use the smallest available volume in keeping with the region of interest. As a trend for the same CBCT unit, the larger the FOV, the greater the effective dose to the patient. However, comparable FOV on different CBCT units produce a range of radiation exposures.

Radiation Considerations in CВCT: Since the introduction of the CBCT in dentistry, there has been considerable discussion and uncertainty about the radiation associated with a CBCT scan. Because of potential hazardous effects of ionizing radiation on living tissues, the radiation exposure to a human being for medical and dental reasons should be kept As Low As Reasonably Achievable (ALARA). Every radiation dose of any magnitude, no matter how small, involves a certain degree of detrimental effect. CBCT effective doses are smaller than those of medical CT. However, significant modifications of those parameters aimed toward significant reduction of the effective dose can reduce the image quality. Therefore, clinical judgment should be exercised with dose reduction efforts in order to maintain diagnostic and quality images.7 It should be noted that the effective doses mentioned here could be rapidly outdated when new CBCT devices come on the market. Therefore, dose measurements of newer equipment should be constantly monitored by an independent agency.

\section{Practical Applications of CBCT in Orthodontics}

Impacted Tooth Position: The most recognized need for CBCT imaging in orthodontics is that of impacted canine evaluation. CBCT imaging is precise in determining not only the labial/lingual relationship but also a more exact angulation of the impacted canine. These 3D images are beneficial in determining the proximity of adjacent incisor and premolar roots, which may be invaluable in determining the convenience of uncovering and bonding. CBCT enhances the power to localize impacted canines accurately, evaluate their proximity to other teeth and structures, determine the follicle size and therefore the presence of pathology, estimate space conditions, assess resorption of adjacent teeth, assist in planning surgical access and bond placement, and aid in defining optimal direction for extrusion of these teeth into the oral cavity.

Root Resorption: CBCT has been shown to be at least as good as periapical radiography for determining tooth and root length. Furthermore, because CBCT can generate precise images of small root defects, it provides more accurate insights into root resorption and has greater sensitivity and specificity than do panoramic or other 2D radiographs in detecting these lesions. Root resorption are often observed readily in CBCT images, and therefore the image clarity allows clinicians to classify the sort of root resorption. For teeth with multiple roots, resorption can be localized to a specific root.

Fractured Roots: To view root fractures radiographically, it's going to be difficult if the fracture is in an oblique direction. With CBCT, the tooth of interest are often viewed altogether the three planes of space making it easier to work out the location of root fracture and degree of displacement.

Orthodontic Implants Placement (TADs): The knowledge of the basic positioning can greatly enhance the chance for correct placement and success of orthodontic implants. CBCT images allow more accurate and dependable views of the inter-radicular relationships than panoramic radiographs. CBCT data are often wont to construct placement guides foe positioning mini-implants between the roots of adjacent teeth in anatomically difficult sites. The volume and quality of the bone within the proposed placement sites are often evaluated before insertion of the mini-implants.

Temporomandibular Joint Assessment: Conventional tomography has been widely used for TMJ evaluation; however, technique sensitivity and therefore the length of the examinations made it a less attractive diagnostic tool for the dentist. CBCT images of the TMJ are shown to supply greater reliability and accuracy than 
tomographic or panoramic views in detecting condylar erosions. Presence of temporomandibular dysfunction can complicate orthodontic treatment and hence requires careful assessment of TMJ anatomy, before, during and after orthodontic treatment. CBCT facilitates visualization of minor to overt osseous hard tissue changes and congruency of articulating surfaces resulting from pathology and adaptive processes and allows for accurate detection and evaluation of pathological changes.

Airway Analysis: Initial investigations on airway patency, function and disorders utilizing CBCT have provided preliminary answers, including dimensions of normal airway anatomy in adults, relationship of 2D to 3D measurements, differences in airway morphology in subjects with OSA and non-OSA, the effects of extractions on 3D pharyngeal volume and structure, and the consequences of RME and orthognathic surgery on airway dimensions.

Cleft Lip and Palate: CBCT can provide the cleft's exact anatomic relationships and bone thickness around the existing teeth in proximity to the cleft or clefts. The information is invaluable for the grafting procedures planned and for possible tooth movement within the existing dentition. CBCT images are valuable for determining the volume of the alveolar defect and, therefore, the amount of bone needed for grafting in patients with CL/P and for determining the success of bone fill following surgery. Finally, CBCT can be useful for diagnosis and treatment of impacted canines that are common in patients with $\mathrm{CL} / \mathrm{P}$ and their paths of eruption through grafted bone sites.

Location of Anatomic Structures: Anatomic structures, like the mental foramen, inferior alveolar nerve, sinus, and adjacent roots are easily visible using CBCT. CBCT images also allow precise measurement of distance, area and volume which helps the clinicians in treatment planning for sinus lifts, ridge augmentations, extractions and implant placements.

Asymmetry Evaluation: Three-dimensional visualization of the patient allows for a more accurate evaluation of both dental and skeletal asymmetries. Presence of a very unilateral crossbite vs one after a shift of the mandible into centric occlusion are often determined more easily by viewing and measuring the maxillary and mandibular bones in three dimensions.

CBCT generated Cephalograms: The CBCT data set can be reformatted to generate a CBCT reconstructed lateral cephalogram so that conventional measurements can be made and compared with existing 2D norms. These CBCT reconstructed lateral cephalograms offer the advantage of ability to digitally reorient the head position in cases in which the patient did not undergo scanning with the proper head position. The image quality also can be enhanced by virtually sculpting away extraneous superimposing skeletal structures. In addition, separate images can be created of the left and right sides for assessment of asymmetries.
Orthognathic Surgery: Several applications of CBCT in orthognathic surgery treatment simulation, guidance and outcome assessment are developed. CBCT 3D surface reconstructions of the jawbones are used for preoperative surgical planning and simulation in patients with traumas and skeletal malformations. Coupled with dedicated software tools, simulations of virtual repositioning of the jaws, osteotomies, distraction osteogenesis and other interventions can now be successfully implemented. indicated where bone information is required in orthognathic surgery planning in order to obtain 3D data sets of the craniofacial skeleton.

Pathologies of Jaws: Presence of radiopaque lesions near the apexes of teeth, like condensing osteitis, dense bone island and focal apical osteopetrosis aren't readily visualized on panoramic radiographs. They appear to have no causative factors but can prevent tooth movement. Such lesions can be viewed easily using CBCT images.

Fabrication of custom orthodontic appliances: The fabrication of custom lingual orthodontic appliances has been demonstrated using CBCT image data with existing technology to virtually plan a patient's treatment and therefore the manufacturing of custom appliances with 3D printing technology (Ye et al., 2011). Such advances appear to be rapid, and that they also promise efficient and effective patient-specific treatments. Correspondingly, Orametrix (Richardson, TX) may be a company that has been using CBCT technology for the last several years to supply the info necessary for planning and executing technology-assisted treatment through its SureSmile system.

Superimpositions: This method eliminates the need for segmentation and selecting landmarks, the errors associated with these steps are eliminated, thereby reducing the overall cumulative errors. Common weaknesses of many outcomes studies are image fidelity and method errors in the superimposition process, leading to confounding and often conflicting results.

Localisation of supernumerary teeth: The localisation of supernumerary teeth specifically when they are multiple is a diagnostic challenge. While single supernumerary teeth could be easily localised using conventional images, cases with multiple supernumerary teeth could benefit from the use of CBCT.

Incidental findings, missed findings and medico-legal implications: The incidence of incidental findings in CBCT images unrelated to the first purpose of the scan are reported to be as high as 25\% during a group of 500 consecutively scanned individuals. These included airway findings, TMJ abnormalities and endodontic lesions. An additional question that requires further study is the capability of the orthodontist to identify non-orthodontically relevant findings and to make appropriate referrals when needed. Lack of the recognition of incidental lesions can have substantial 
medicolegal ramifications. In contrast, the potential for inadvertent diagnosis of false-positive findings by the untrained eye have the potential to add unnecessary costs to healthcare, as well as cause unnecessary anxiety to the patient and family.

Advantages of CBCT Imaging in Orthodontics: CBCT imaging provides many unique features and advantages to orthodontic practice over conventional extraoral radiographic imaging.

- Greater Clinical Imaging Efficiency. Full field of view (FOV) CBCT imaging provides volumetric data acquisition of the entire maxillofacial skeleton in a single radiographic procedure. This data can be easily reformatted to provide simulated radiographic (e.g. lateral and posterior-anterior cephalometric, panoramic) or comparable volumetric images currently used in orthodontic diagnosis, cephalometric analysis, and treatment planning. This provides for greater clinical imaging efficacy

- Provides 3D Visualisation. CBCT data can be reconstructed to provide unique, inter-relational, orthogonal (i.e. axial, sagittal and coronal) images previously unavailable for orthodontic assessment. Other imaging processing can also be performed such as maximum intensity projection (MIP) and surface or volumetric rendering that provides an interactive volumetric model enabling visualisation and interrelationship of craniofacial structures including the maxillofacial skeleton and soft tissue boundaries like the airway and facial outline.

- Anatomic Accuracy. Unlike traditional extraoral and panoramic radiography, CBCT images and renderings are anatomically accurate and can be displayed at any angle for any part of the maxillofacial skeleton. Therefore, actual measurements can be obtained without the need for considerations of magnification or projection discrepancies.

Limitations and Risk considerations of CBCT: CВCT images have inherent "noise" that reduces image clarity and produces limited soft tissue contrast resolution. CBCT image quality is also affected by image artifacts such as streaking, shading, rings, and distortion due to high areas of attenuation (such as metallic restorations) and inherent spatial resolution may limit adequate visualisation of structures in the dento-alveolar region. CBCT imaging uses ionising radiation which is a potential carcinogen.

Recent public and scientific reports have increased public awareness and professional concerns over the potential association between diagnostic radiation exposure and cancer. The risk associated with various dental radiographic procedures, specifically CBCT imaging, has most often been compared to the radiation dose imparted by a "baseline" imaging modality (e.g. typical panoramic radiographic procedure) or to average background equivalent radiation time (e.g. days of background). The use of patient protective shielding like lead torso aprons and thyroid shields. is recommended, when possible, to minimise exposure to radiosensitive organs outside the field of view. The use of low dose protocols may be adequate for low level diagnostic tasks such as root angulations.

Future uses for CВCT: Technology is consistently changing and new applications arise almost daily. the subsequent applications of CBCT technology gives a glimpse of what could also be available within the near future.

- Virtual Models CBCT data are often wont to produce 3D digital study models without the necessity for alginate impressions. It avoids patient discomfort and saves orthodontist's valuable chair time. These models are of upper diagnostic value than other digital models because it includes not only the tooth crowns but also roots, impactions, developing teeth and alveolar bone.

- Invisalign Aligner it's going to be possible within the future to execute the whole fabrication process of the aligners using CBCT digital data. The CBCT images might be wont to create the virtual models, thus negating the necessity to require and mail impressions and bite registration. This information is often transferred electronically to laboratories and therefore the desired virtual tooth movement are often accomplished by way of e-mail communication between the orthodontist and the laboratory. Even the retainers might be fabricated by the info within the laboratory electronic database of the ultimate tooth positions.

- Indirect Bonding of Brackets Construction of 'hardcopy' models from the CBCT image are often used for laboratory procedures required for indirect bonding

- Custom-made Brackets and Wires CBCT data are often used precisely to manufacture custom made orthodontic brackets and wires for a private patient.13

- In the future, it's likely that superimposition or similar approaches will replace or complement linear and angular measurements made on images from 3D.

- Segmentation of bone, teeth or the other structure from CBCT scan, are going to be achieved automatically, saving time and avoiding bias. An equivalent is going to be for anatomic landmarks and measurements through the synthetic intelligence and computer vision. CBCT imaging of digital models are going to be an alternate to digitization with optical devices, thus a further dedicated optical dental digitizer wouldn't be necessary required.

- Individualized brackets, and arch wires bent by robots are going to be fabricated supported these digital casts. within the next few decades, a customized treatment and biomechanical planning are going to be more fully realised, maybe in one's own office. 


\section{CONCLUSION}

Three-dimensional CBCT based hard and soft tissue simulations, photographic integrations and superimpositions have ushered a new era of dynamic CBCT imaging. Future developments during this field offer promises of even greater benefits in orthodontic diagnosis and treatment. The long-awaited incorporation of the 3D to our radiographic records is soon becoming a reality. CBCT is the way forward for orthodontics and therefore the applications in orthodontics seem almost limitless. the recognition of CBCT imaging in orthodontics is probably going to extend significantly within the future. Currently, the limiting factor for widespread use of CBCT in orthodontics is the radiation dose especially in children. Further technical development of CBCT devices and flat panel detectors will reduce the radiation dose within the future and fully adjustable collimation of the radiation beam to the specified field of view (FOV) will contribute to lowering the radiation dose.

\section{REFERENCES}

Anne Marie Kuijpers-Jagtman; Mette A.R. Kuijpers; Jan G.J.H. Schols; Thomas J.J. Maal; Karel H. Breuning; Olivier J.C. van Vlijmen The use of cone-beam computed tomography for orthodontic purposes Seminars in Orthodontics, ISSN: 1073-8746, Vol: 19, Issue: 3, Page: 196-203

Abdelkarim, Ahmad. (2015). Myths and facts of cone beam computed tomography in orthodontics Abdelkarim.

Abdelkarim, Ahmad. "Cone-Beam Computed Tomography in Orthodontics." Dentistry journal vol. 7,3 89. 2 Sep. 2019, doi:10.3390/dj7030089

Agrawal JM, Agrawal MS, Nanjannawar LG, Parushetti AD.( 2013) CBCT in Orthodontics: The Wave of Future. J Contemp Dent Pract;14(1):153-157.

Coskun, Ipek, and Burçak Kaya. (2018) "Cone Beam Computed Tomography in Orthodontics." Turkish journal of orthodontics vol. 31,2: 55-61. doi:10.5152/ TurkJOrthod.2018.18020
European Commission (2012). Radiation protection No 172: cone beam CT for dental and maxillofacial radiology. Evidence based guidelines.

Kapila S, Conley RS, Harrell WE Jr. The current status of cone beam computed tomography imaging in orthodontics. Dentomaxillofac Radiol.;40(1):24-34. doi:10.1259/dmfr/12615645

Leonardi R. (2011) Cone-beam computed tomography and three-dimensional orthodontics. Where we are and future perspectives. J Orthod. 2019;46(1_suppl):45-48. doi:10.1177/1465312519840029

Machado, Genevive L. (2015) "CBCT imaging - A boon to orthodontics.” The Saudi dental journal vol. 27,1: 12-21. doi:10.1016/j.sdentj.2014.08.004

Mah JK, Huang JC, Choo H.( 2010) Practical applications of cone-beam computed tomography in orthodontics. J Am Dent Assoc.141 Suppl 3:7S-13S. doi:10.14219/jada. archive.2010.0361

Makdissi J. Cone beam CT in orthodontics: the current picture. Int Orthod. 2013;11(1):1-20. doi:10.1016/j. ortho.2012.12.011

Parveen, Shahista \& Kulkarni, Uma \&t Mascarenhas, Rohan \&t Shetty, Roopali. (2019). Awareness and Practice of Ethics and Guidelines with Cone-Beam Computed Tomography Prescription in Orthodontics. Journal of Indian Orthodontic Society. 53. 49. 10.4103/jios. jios_134_18.

Scarfe WC, Azevedo B, Toghyani S, Farman AG. (2017) Cone Beam Computed Tomographic imaging in orthodontics. Aust Dent J. 62 Suppl 1:33-50. doi:10.1111/adj.12479

Tadinada, Aditya \& Schneider, Sydney \& Yadav, Sumit. (2018). Role of Cone Beam Computed Tomography in Contemporary Orthodontics. Seminars in Orthodontics. 24. 10.1053/j.sodo.2018.10.005.

Van Vlijmen OJ, Kuijpers MA, Bergé SJ, et al.( 2012) Evidence supporting the use of cone-beam computed tomography in orthodontics. J Am Dent Assoc. 143(3):241-252. doi:10.14219/jada.archive.2012.0148. 